\title{
MOVIE RECOMMENDATION SYSTEM
}

\author{
Darshini M \\ Department of CSE \\ Vidyavardhaka college of engineering, \\ Mysore, Karnataka, India \\ Rakshit Mysore Lokesh \\ Department of CSE \\ Vidyavardhaka college of engineering, \\ Mysore, Karnataka, India
}

\author{
Abishay Raina \\ Department of CSE \\ Vidyavardhaka college of engineering, \\ Mysore, Karnataka, India \\ Mohammed Noorulla Khan Durrani \\ Department of CSE \\ Vidyavardhaka college of engineering, \\ Mysore, Karnataka, India
}

\begin{abstract}
Recommender system is the technique which helps the users to fetch usefull and required data according to their interests and preferences by filtering large amount of information. Over the past few years, many recommendation systems for different domains have been developed. Entertainment is one of the significant domain as it is necessary for each one of us to refresh our mood and energy. Movie recommender systems helps users to automatically choose preferred movies from a huge collection. To implement this system, collaborative filtering technique is most widely used as it is more advantageous than other techniques. But this technique also suffers from many issues such as, cold start, data sparsity, shilling attack etc. Many methods have been developed to solve these issues.
\end{abstract}

Keywords - Movie recommendation system, collaborative filtering, KNN clustering, Pearson correlation, User's psychological profile.

\section{INTRODUCTION}

Due to the information overload and popularity in social media, Recommender systems are being explored and developed continuously. Based on its wider usability in social media, recommendation systems are mainly classified into six categories. They are domains, datasets, data mining methods, types of recommendation and performance metrics. But the research of recommender system is focussed more on entertainment domain (mainly movies). This is due to the change in the taste and preferences of users day by day and also easily and publicly available datasets in this domain by research community.
Movie recommender engine can be implemented mainly using content based, collaborative filtering or hybrid methods. Collaborative filtering method has more benefits over other methods. This filtering technique considers past history and interests of other similar users to recommend the current user. So that it improves the performance and efficiency of the recommender system. Though hybrid method gives better results, it is quite expensive and complex compared to collaborative approach. Collaborative filtering also suffers from many issues. The problem of cold start is one among them. The prediction of recommendations to the new user by considering new item is one of the most challenging issue. Recommendations can be predicted for the active user on the basis of known ratings of movies which are already given by a user. For a new user who is registered for the first time in the system has no ratings and known only demographic details of a user. Hence, there is a huge scope of exploration in movie recommendation systems for improving scalability, accuracy and quality of recommendations given to new users.

\section{LITERATURE SURVEY}

There are mainly two types of collaborative filtering. They are memory based and model based methods. The first one recommends movies to users based on their past data and the other one implements some model to recommend movies to users. Memory based method is preferred over other methods due to it's easy implementation and it is also less expensive. Memory-based method computes the recommendations which are given to the users by considering the whole data set.

It makes use of similarity measures to select users or movies which are similar to the active user. Recommendations are then calculated by using the ratings of these neighbours or groups. Memory-based filtering method is again divided into 


\section{International Journal of Engineering Applied Sciences and Technology, 2019 \\ Vol. 3, Issue 11, ISSN No. 2455-2143, Pages 39-41 \\ Published Online March 2019 in IJEAST (http://www.ijeast.com)}

user-based and item-based filtering methods (Patel et al., 2017).

Clustering algorithms are most commonly used to partition the set of movies on the basis of user rating data. In clustering, $\mathrm{K}$ Nearest Neighbour (KNN) method is widely used because of it's simple implementation. Furthermore, it can be easily understood and explained. The interaction between user and item is categorized into two ways. One is Implicit interaction which involves sessions and cookies of browser, other one is explicit interaction which involves ratings and feedback given by the user (Anandhan et al., 2018).

Similarity among users is calculated using Pearson correlation coefficient to get optimal results when compared to other techniques. The asymmetric method explains that similarity of user $\mathrm{B}$ with $\mathrm{A}$ is different than the similarity of $\mathrm{A}$ with $\mathrm{B}$. To make better prediction of recommendations, Linear Regression method is employed (Katarya et al., 2016).

A variety of performance metrics can be used to evaluate the predictions generated by recommender systems in social media. It is observed that the highly used evaluation metrics are precision and recall, which are popular with recommender systems in social media and also plays important role in information retrieval.

To solve the issues of new user and new item, a lot of methods have been implemented. A method involving tags and keywords to provide information about user and item is computed (Hande et al., 2016). In one of the content based filtering technique, the psychological profile of a user was employed. This profile can be identified with the help of a yes/no psychological test. The test was carried out by posing different queries regarding individual's personality.

The objective of this test was to identify to what degree the four main psychological profiles such as sanguine, choleric, melancholic and phlegmatic can be found in the user's own personality. The main aim of this method is to observe if there is a similarity between a persons' psychological profile and his/her preferences in movies and to recommend movies specifically that suits the user (Costin-Gabriel et al., 2015).

To capture the user interests, temporary features of a user profile is used. This feature constitutes the user interests and can be varied over time. The temporary feature is computed using time bins, where each bin representing a group of movies that are rated at the same time slice. Context-aware recommender system involves contextual information such as location, time and social data to compute recommendations (Bagher et al., 2017).

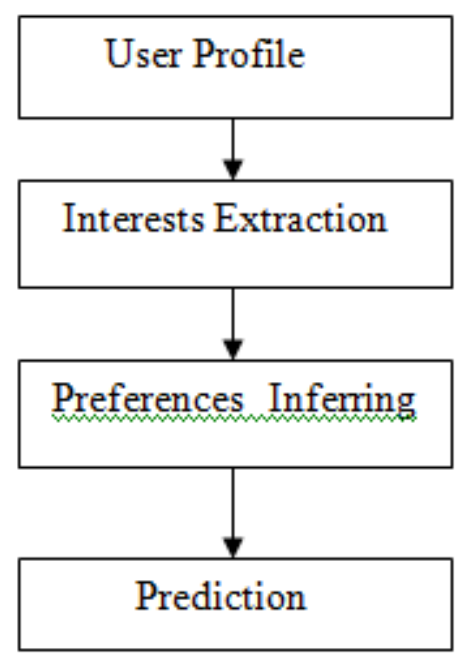

Fig. 1. Recommendation from User profile.

In one of the method to solve cold start problem, Association rule and clustering techniques are combined, where association rule is applied to the sparse profile of a new user to obtain enriched profile and clustering technique is applied to the sparse ratings of new items. Then the combined result is used to predict recommendations (Hridya et al., 2013).

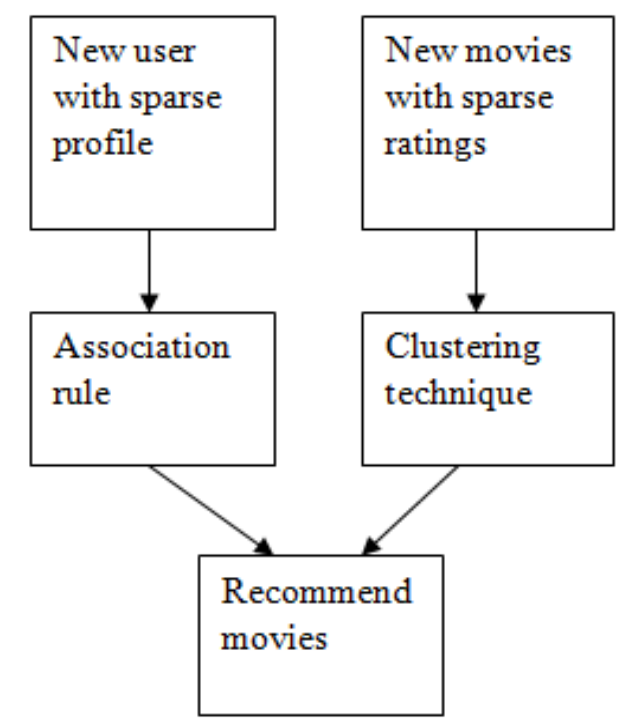

Fig. 2. Combination of Association rule and clustering technique.

One more interesting challenge of collaborative filtering technique is shilling attack, which means that some malicious users can create fake profiles and insert into the network in 


\section{International Journal of Engineering Applied Sciences and Technology, 2019 \\ Vol. 3, Issue 11, ISSN No. 2455-2143, Pages 39-41 \\ Published Online March 2019 in IJEAST (http://www.ijeast.com)}

such a way that it affects the recommender system. To reduce this, various trust calculation techniques are used. One of them is the Novelty method, which adds different weights on the social trust relationships among users based on the trustee's competence and trustworthiness (Katarya et al., 2016). This method can make sure that recommendations always comes from the trusted users.

\section{CONCLUSION}

In this survey, it analyses the current state of development in movie recommender systems. The optimal way of implementing movie recommender system is studied. Though Collaborative filtering technique is widely used and implemented, it also suffers from many issues. The main issues such as new user and new item problem, shilling attack are discussed. Various methods to reduce these problems are also depicted. The future enhancement of this paper is to compute better recommendations to new users by combining existing methods along with individual's social media details.

\section{REFERENCE}

[1] Lakshmi Tharun Ponnam, Sreenivasa Deepak Punyasamudram, Siva Nagaraju Nallagulla, Srikanth Yellamati. (2016). "Movie Recommender System Using Item Based Collaborative Filtering Technique", IEEE.

[2] Yuri Stekh, Mykhoylo Lobur, Vitalij Artsibasov, Vitalij Chystyak. (2015). "Methods and Tools for building Recommender system" - CADSM 2015, 24-27 February, UKRAINE.

[3] Rahul Katarya, Om Prakash Verma.(2016). "Effective collaborative movie recommender system using asymmetric user similarity and matrix factorization", International Conference on Computing, Communication and Automation(ICCCA2016).

[4] Shreya Agrawal, Pooja Jain. (2017). "An Improved Approach for Movie Recommendaton System". International Conference on I-SMAC( IOT in Social, Mobile, Analytics and Cloud)(I-SMAC 2017).

[5] Bansari Patel, Palak Desai, Urvi Pancha. (2017). "Methods of Recommender System: A Review" - 2017 International Conference on Innovations in Information Embedded and Communication Systems(ICIIECS).

[6] M V V R Murali Krishna Rao. (2017). "A Collaborative Filtering Recommender System with Randomized Learning Rate and Regularized Parameter".

[7] Soanpet .Sree Lakshmi, Dr.T.Adi Lakshmi. (2014). "Recommendation System: Issues and Challenges",
(IJCSIT) International Journal of Computer Science and Information Technologies, Vol. 5 (4) .

[8] Bei-Bei CUI. ( 2017). "Design and Implementation of Movie Recommendation System Based on Knn Collaborative Filtering Algorithm", ITM web of conferences.

[9] Ningning Yi, Chunfang Li, Xin Feng, Minyong Shi. (2017). "Design and Implementation of Movie Recommender System Based on Graph Database". 2017 14th Web Information Systems and Applications Conference.

[10] Anitha Anandhan, Liyana Shuib, Maizatul akmar ismail and Ghulam mujtaba.(2018). "Social Media Recommender System: Review and Open research issues" 2018 The University of Malaya's Research Grant Programme SBS (Equitable Society) under Grant RP059E-17SBS.

[11] Costin-Gabriel Chiru, Vladimir-Nicolae Dinu, Catalina Preda,Matei Macri. (2015). "Movie Recommender System Using the User's Psychological Profile" 2015 IEEE.

[12] Gaurav Arora, Ashish Kumar, Gitanjali Sanjay Devre, Prof. Amit Ghumare. (2014). "movie recommendation system based on users' similarity" 2014 International Journal of Computer Science and Mobile Computing.

[13] Rupali Hande, Ajinkya Gutti, Kevin Shah, Jeet Gandhi, Vrushal Kamtikar. (2016). "moviemender - a movie recommender system" 2016 International Journal of Engineering Sciences \& Research Technology.

[14] Steven Postmus. (2018). "Recommender System technique applied to Netflix movie data" Reasearch Paper Business Analytics,Vrije Universiteit Amsterdam, February 2018.

[15] Bagher Rahimpour Cami, Hamid, Hassanpour Hoda Mashayekhi. (2017). "A Content-based Movie Recommender System based on Temporal User Preferences" 2017 3rd Iranian Conference on Signal Processing and Intelligent Systems (ICSPIS).

[16] Hridya Sobhanam, A.K.Mariappan. (2013). "Addressing cold start problem in recommender systems using association rules and clustering technique" 2013, IEEE. 